\title{
Ramifications of Machine Learning in the Manufacturing Sector
}

\author{
Ashish Gupta \\ JSS Science and Technology University \\ ashishgupta63966@gmail.com
}

\begin{abstract}
The manufacturing sector in present India faces more complex, dynamic and chaotic behaviour to satisfy the demand for high quality products in an efficient manner. Machine learning has been around for some decades now, but its impact and significance in this domain is still impugned. The two sectors, though being ends apart, surprisingly have the potential to collaborate and enhance performance and productivity of the end-result manufacturing. Experimental analysis and research using machine learning algorithms with manufacturing under consideration has been ongoing and the results undoubtedly prove that the combination of the two sectors will be inevitable in the near future. The fact that machine learning is data-driven and prediction is its sub-domain enables the smooth introduction of manufacturing in its grasp. This paper provides insight to how machine learning could disrupt the manufacturing development in India and could result in better production yield and revenue generation. In the next 20 years, automation would be a major factor in yielding profits to large manufacturing corporations and industries and machine learning would have an immense role to play in the rapidly changing scenario.
\end{abstract}

Keywords: Machine Learning, Feature extraction, Prediction, Manufacturing

\section{Introduction}

Machine learning enables computing devices and machines to automatically improve their performance at some tasks through procedural algorithms. Machine learning approach to manufacturing is new and this form of technological innovation has massive potential to cause exponential enhancement in conventional methodologies. From striving to keep supply chains operating efficiently to producing customised, built to order products on time, machine learning algorithms have the potential to bring greater predictive accuracy to every phase of production.

\section{Contribution to GDP}

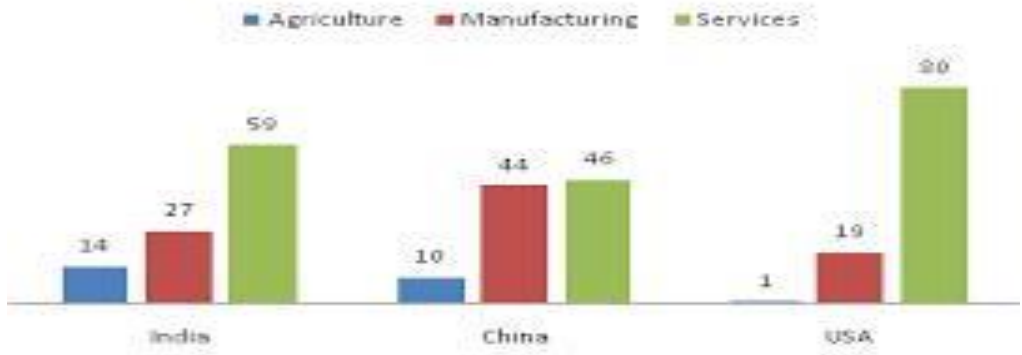

Figure 1. GDP sector composition graph 


\section{Gyancity Journal of Engineering and Technology, Vol.3, No.1, pp. 29-35, January 2017 \\ ISSN: 2456-0065 DOI: 10.21058/gjet.2017.31005}

Many of the algorithms being developed are iterative, designed to learn continually and seek optimised outcomes. These algorithms iterate in milliseconds, enabling manufacturers to seek optimised outcomes in minutes versus months. The Make in India call focuses on India becoming a manufacturing hub where the Prime minister Narendra Modi intends to improve the ease of doing business. Manufacturing or industry sector does not create millions of jobs proportionally in terms of investment unlike the services sector. Hence this sector needs enhanced upgradation in technology or huge investment from global companies to setup factories in India if it has to compete against China or USA. Machine learning is the right technology upgradation needed to blend into the Indian manufacturing scenario. The advent of algorithms in quality control of industrial designs would yield in better production and more exports.Machine Learning algorithms can be implemented in various stages in a production unit. This paper identifies and elucidates the outward quality control stage in a manufacturing process. The basic principle is to lower the error in manufacturing of industrial parts or components with comparison to ideal model components. Machine learning algorithms can be implemented in order to determine the anomalies in a newly manufactured component and verifying the quality with respect to a dataset from an already obtained ideal component. This procedure locates anomalies and also is capable of performing predictive analysis on different manufacturing defects using certain features for identification.

\section{Feature Extraction and Matching}

In manufacturing, the components are susceptible to dents or uncalled defects that can occur due to machinery. This can result in performance loss in the overall product. Hence each component being manufactured is subjected to machine learning algorithms by extraction of their features using a CAD based model. For interpretation, the CAD model is broken down into single surfaces and for each of these surfaces, the fundamental curvature characteristics are derived and stored. or each surface we output the surface ID, the surface type and the curvature characteristics. This information forms the training data set. The sensor data is obtained by 3D mapping of component using range image segmentation. A manufactured component under test typically consists of several individual surfaces. A routine is implemented which iterates over all surfaces of the model. These results are tabulated which forms the test dataset. 3D mapped range image segmentation of the test component is obtained. The industrial part under test is subjected to feature extraction with respect to curvature and shape classification along with the surface ID of each surface. This system proposes a modeldriven approach for feature extraction as shown in Figure 2. Machine learning algorithms are implemented with the training dataset and the test data for each surface to check if the output surface ID is found to be matching. The matching of test component dataset using range image segmentation with the ideal CAD model would help with in identification of the features which are erroneous or have abnormalities. 


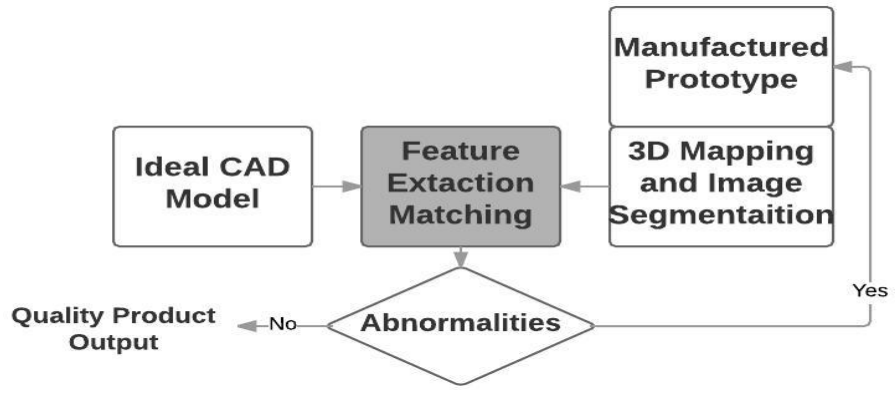

Figure 2. Feature matching of industrial component with CAD design

\section{Machine Learning Implementation}

Apart from determining the algorithm validation and testing, another important procedure is to determine which algorithm is to be used and for what purpose. Machine Learning is a wide concept that is generically labelled and interpreted. The application context and scenario of implementation needs to be given almost importance. The following algorithms can play a vital role in impacting the manufacturing sector.

\subsection{Unsupervised Anomaly Detection Algorithm}

Anomaly detection, also called fraud detection, can be used in the manufacturing domain for identification of manufacturing defects and anomalous parts used in production. The term unsupervised here is used to depict that the output expected value is unknown. There are a finite set of features and anomalous features are obtained only with the help of the given set of data. Consider $\mathrm{x} 1$ and $\mathrm{x} 2$ to be two features like curvature and surface area in a particular manufacturing component. There are $\mathrm{m}$ such samples with curvature and surface area descriptions and a graph as shown below is plotted.

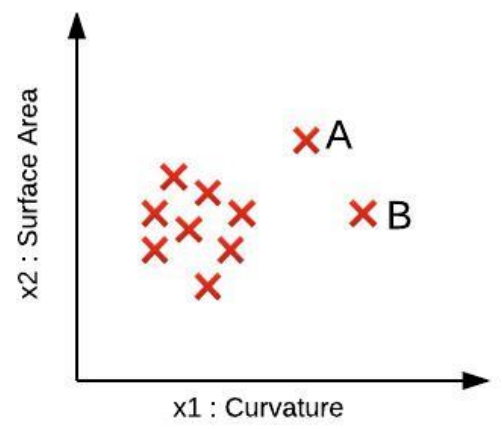

Figure 3. Anomaly Detection: Here, A and B are considered anomalies

There are certain cases in the graph say points such as A and B which are much deviated from the average set of points which are assumed to be normal. The shifted points are considered abnormal points and are identified explicitly. This application is useful in manufacturing applications to dent defects and shape distortions. 


\section{Gyancity Journal of Engineering and Technology, Vol.3, No.1, pp. 29-35, January 2017 \\ ISSN: 2456-0065 DOI: 10.21058/gjet.2017.31005}

\subsection{Prediction in Manufacturing}

Prediction can be a very useful tool when analysis of manufacturing essentials is concerned. In this context, a manufactured component's success rate can be determined with the help of already obtained samples of data from previous manufactures. Suppose a manufactured aircraft engine's success rate is to be determined with the help of training samples collected from previous 1000 engines manufactured. Each engine comprises of varied parameters for development, say size of the engine and the fuel intake be considered as some of many parameters and the performance of each of the 1000 samples is obtained, whether they turned out to be successful or not. This forms the training dataset. Now, when a new engine is manufactured, according to the specification of the two parameters of the new engine, predictive analysis can be done if this engine can be classified as successful using the training dataset. In this example of aircraft engines, the binary classification logistic regression algorithm can be applied. This is a supervised learning algorithm where the possible output results are known. This estimates the probability that a characteristic is present given the values of explanatory variables. Here, the output vector is 0 or 1 , not successful or successful, where 0 is usually taken as the negative class and 1 as the positive class. The real number from the dataset is to be mapped to $(0,1)$ interval, making it useful for transforming an arbitrary-valued function into a function better suited for classification.

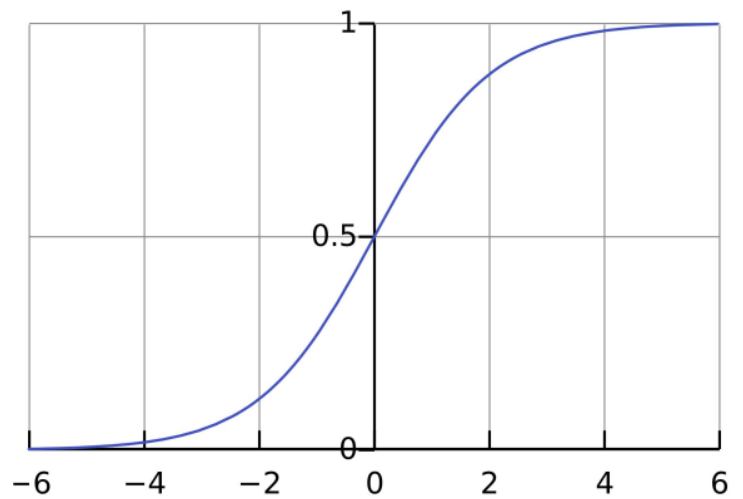

Figure 4. Sigmoid Function Curve for Binomial Logistic Regression

This is done by using a sigmoid function for a binary logistic regression is as shown in figure 4 above. For an ideal application, all predictions greater than 0.5 is set as 1 else as 0 . The $y$ axis will give the probability that the output is 1 . For example, 0.7 gives us $70 \%$ probability that our output is 1 .

\subsection{Multinomial Cluster Classification using ANN}

Artificial Neural Networks(ANN) form an effective iteration of the machine learning concept. There can be multiple possible outputs that need to be envisioned when performing manufacture analysis. This can be done using Linear Vector Quantisation (LVQ) algorithm which is a special type of ANN. The algorithm is a prototype based supervised classification algorithm which is represented by prototype weights which are defined in the feature space of the ob-served data. This algorithm is used to train the obtained dataset and identify the different classifiers clusters or 


\section{Gyancity Journal of Engineering and Technology, Vol.3, No.1, pp. 29-35, January 2017 \\ ISSN: 2456-0065 DOI: 10.21058/gjet.2017.31005}

outcomes so that when a new test dataset is provided, the system identifies the closest matching classifier outcome. Graphically, the euclidean distance between the input test vector and the centroid weights of the trained dataset is obtained and the classifier with the least distance is considered and that is identified as the possible output. The neural network undergoes iterations until the classifiers are distinct. The weights of the clusters in neural network are displaced as the training of dataset takes place. With respect to industrial manufacturing, like the CAD model comparison with the respective industrial component, LVQ can be used to cluster the different surfaces as the data progresses. This is described in the following image. Note how the weight for each cluster of data moves with respect to the input data set of the same category as the training progresses.

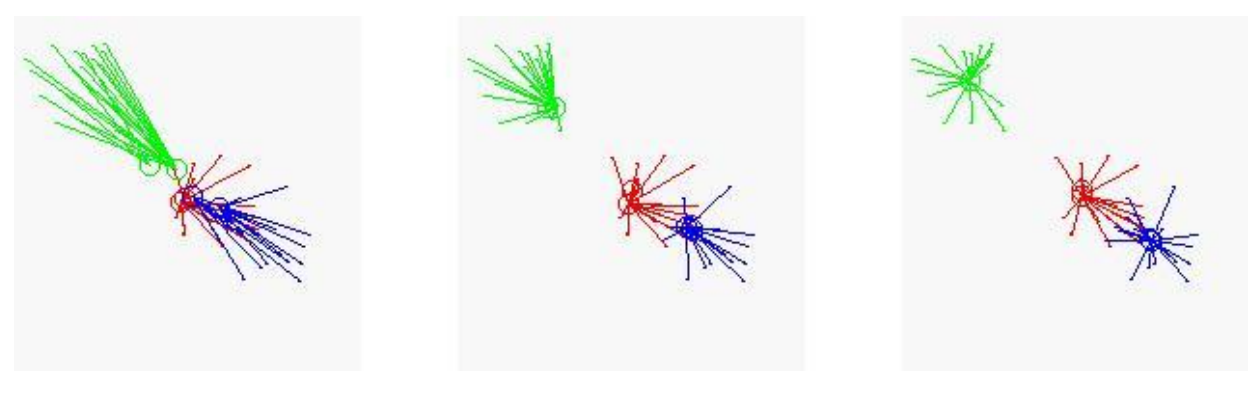

Figure 5. Linear Vector Quantisation (LVQ) ANN representation

\section{Reliability Issue and Limitations of Machine learning}

Despite the understanding that the collaboration of manufacturing and machine learning follow great compatibility, there needs to be more experimental analysis and research to recon the practical benefits of such a mix. Reliability of machine learning algorithms can be put into question as a small error in the system design would be disastrous to large scale industrial manufacturing and companies would incur massive losses. So this paper realises the merits and demerits of applying machine learning to manufacturing.

\subsection{Advantages of Machine learning}

- Automation: A given task undergoes good feature representations and the system itself randomly initialises and trains on the dataset. This is one of the most interesting features. Making sense of unbiased random data is possible now with sophisticated algorithms and hardware to support them.

- Optimisation of parameters: Optimal setting of parameters for visualising large datasets is not feasible for a human. Algorithms can determine the best parameters to consider in an application which can run on a priority basis to predict the best required results. 


\section{Gyancity Journal of Engineering and Technology, Vol.3, No.1, pp. 29-35, January 2017 \\ ISSN: 2456-0065 DOI: 10.21058/gjet.2017.31005}

- Self Modifying: Faster implementations of algorithms can be made possible as little tweaks in the algorithms enable the system to modify automatically to meet the time demands as manufacturing is time dependent to produce quality products in lesser time.

- Multiple Iterations: In manufacturing, the scale of realisation of industrial components is large, hence conventional methods of quality assurance would take a long time. Applications of machine learning would implement multiple iterations of realising the dataset thus speeding the process.

\subsection{Disadvantages of Machine learning}

- Limited Functionality: Machine learning may not work in every case imaginable. Proper understanding of the application is required to determine the methodology used by machine learning implementation.

- Large data requirement: A small dataset may prove to cause errors in the functioning of the system since different probable cases would be missing. It might be clumsy to work with and collect such large amounts of data.

Hence, the fact remains that machine learning is just a tool and it depends on the user to determine how it is implemented. The manufacturing applications are to be fully understood and suitable machine learning techniques need to be applied for higher efficiency and productivity.

\section{Conclusions}

This paper proposes how ideal CAD models can be compared with industrial components and determine abnormalities, dents and uncalled errors in production, using feature extraction from various parameter. This paper also highlights different machine learning algorithms and how each would impact in better outcomes. Prediction analysis also forms a major chunk of machine learning in the manufacturing domain to provide sustained growth. The paper assimilates these concepts and elucidates on how this would disrupt the conventional systems in place. Adoption of machine learning in heavy industrial settings is still very limited, largely due to its recent emergence as a viable control method. Different industry segments are at different positions on the adoption curve. Machine learning's core technologies align well with complex problems manufacturers face daily. This would result in the revolutionising product and service quality, increase in production yields by the optimisation of team, machine, supplier and customer requirements. Machine learning is ideally suited for optimising supply chain and creating greater economies of scale. Machine learning algorithms have paved way which can help manufacturers bring about operational and business transformation leading to increased accuracy in decision making and improved performance. 


\section{Acknowledgements}

I am grateful to Prof. M N Shanmukha Swamy (JSS Science and Technology University), Sandesh Jagadish (JSS Science and Technology University), Ritwick Medikeri (University of Michigan, Ann Arbor), and Prof. Sriram Kalyanaraman (University of Florida) for helpful reviews of a draft of this paper.

\section{References}

[1] "Using Big Data for Machine Learning Analytics in Manufacturing" By Jiby Joseph, Omar Sharif, Ajit Kumar, Saurabh Gadkari and Aditya Mohan, Manufacturing Innovation and Transformation Group, TCS

[2] "Machine learning in manufacturing: advantages, challenges, and applications" by Thrsten Wuest, Daniel Weimar, Christopher Irgens

[3] "The Discipline of Machine Learning" By Tom M. Mitchell, Carnegie Mellon University, PA, 2006

[4] "Machine Learning Approaches for Failure Type Detection and Predictive Maintenance" By Patrick Jahnke, Technische Universitat Darmstadt, 2015

[5] "Machine-learning techniques and their applications in manufacturing" D.T Pham , University of Birmingham

\section{Author}

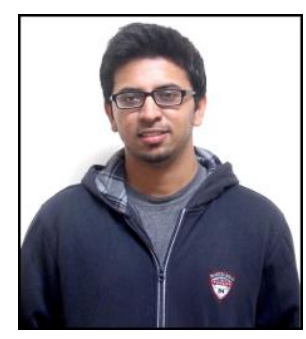

\section{Ashish Gupta}

A student of Electronics and Communication stream, Ashish Gupta is pursuing his BE degree in the autonomous JSS Science and Technology University, Mysore. 